\title{
Dynamical analysis of a weakly coupled nonlinear dielectric waveguide: Surface-plasmon model as another type of Josephson junction
}

\author{
Yasa Ekşioğlu, ${ }^{*}$ Özgür E. Müstecaplığlu, and Kaan Güven \\ Department of Physics, Koç University, Istanbul, Turkey \\ (Received 11 June 2011; published 6 September 2011)
}

\begin{abstract}
We propose that a weakly coupled nonlinear dielectric waveguide surface-plasmon system can be formulated as another type of Josephson junction. Such a system can be realized along a metal-dielectric interface where the dielectric medium hosts a nonlinear waveguide (e.g., fiber) for soliton propagation. We demonstrate that the system is in close analogy to the bosonic Josephson junction of atomic condensates at very low temperatures, yet exhibits different dynamical features. In particular, the inherently dynamic coupling parameter between soliton and surface plasmon generates self-trapped oscillatory states at nonzero fractional populations with zero and $\pi$ time-averaged phase difference. The salient features of the dynamics are presented in the phase space.
\end{abstract}

DOI: 10.1103/PhysRevA.84.033805

PACS number(s): 42.65.Tg, 42.65.-k, 05.45.-a, 03.75.Lm

\section{INTRODUCTION}

Plasmonics encompasses the science and technology of plasmons, which are the collective oscillations of electrons mainly in metals. In particular, the coupling of plasmons with photons gives rise to a hybrid quasiparticle known as the surface plasmon-polariton (also called surface plasmon) [1], which can propagate along metal surfaces. As a result, light can be coupled to and propagate through structures that are much smaller than its wavelength. Thus, plasmonics enables the subwavelength photonics [2,3], bridges it to electronics at nanoscale, and offers promising applications in nano-optics and electronics, such as lasing and sensing [4-7].

In this context, the coupling between the surface plasmonpolaritons and the dielectric waveguide modes is of interest for integrated optoelectronic applications. Much effort has been devoted to understand and enhance this coupling in guided mode geometries [8-10] and in layered systems [11]. In particular, a recent work describes the resonant interaction model between the copropagating surface plasmons on a metal surface and solitons in a nonlinear dielectric medium [12]. In that model, the interaction depends on, and hence can be controlled by the soliton amplitude, which leads to coupled surface plasmon-soliton modes, and provides a way of manipulating the surface plasmon propagation. Motivated by this study, we propose in this paper that a weakly coupled nonlinear dielectric waveguide surface-plasmon system can be formulated as a type of dielectric waveguide surface-plasmon Josephson junction (DWSP-JJ) and investigate its dynamics within the model introduced in Ref. [12]. This formulation enables a remarkable connection between this optical system and the well-known Josephson-Junction dynamics of BoseEinstein atomic condensates [13] and opens a perspective to quantum plasmonics, where quantum optical phenomena can be realized and utilized via coupling to surface plasmons [14].

The paper is organized as follows: In Sec. II, the theoretical model is introduced. Section III investigates the dynamical features of the DWSP-JJ system in comparison to superconducting Josephson junction (SJJ) and bosonic Josephson junction (BJJ) systems. Section IV concludes the paper.

*yeksioglu@ku.edu.tr

\section{MODEL AND THEORETICAL FORMULATION}

Within the scope of this paper, we adopt the model system given in Ref. [12]. This intuitive model consists of the copropagating (nonlinear) optical soliton and (linear) surface-plasmon (SP) electric fields along a metal-dielectric interface. The nonlinearity in the dielectric is assumed to be confined at a distance $d$ from the metal interface such that the surface-plasmon propagation retains linearity, and the weak coupling between the soliton and SP fields can be treated perturbatively. We note that one can also consider nonlinear SP propagation with the nonlinearity being present along the interface or by other mechanisms. This is an interesting problem in itself and it has been shown theoretically that the nonlinearity would result in self-focusing of surface plasmons and the formation of surface-plasmon solitons $[15,16]$.

Assuming translational invariance in the $z$ direction, and choosing $y$ as the propagation direction, the total electric field of the coupled system is written in the form,

$$
E(x, y)=c_{p}(y) e^{-\kappa_{p} x}+\frac{c_{s}(y)}{\cosh \left[\kappa_{s}(x-d)\right]},
$$

where $c_{p, s}(y)$ are the surface-plasmon and soliton amplitudes, multiplied by the respective transverse profiles with $\kappa_{p}=$ $\sqrt{k_{p}^{2}-k^{2}}$ and $\kappa_{s}=k \sqrt{\gamma / 2}\left|c_{s}\right|$. The propagation wave vector is $k$ whereas $k_{p}$ is the surface-plasmon wave vector. The nonlinearity parameter of the dielectric medium is $\gamma$. This product ansatz for the lateral and longitudinal field profiles reduces the system to essentially a one-dimensional problem, where the amplitudes $c_{p, s}(y)$ obey the following coupled oscillator equations, which are written in the dimensionless coordinate $\xi=k y$ :

$$
\ddot{C}_{p}+\beta_{p}^{2} c_{p}=q\left(\left|c_{s}\right|\right) c_{s}, \quad \ddot{C}_{s}+\beta_{s}^{2} c_{s}=q\left(\left|c_{s}\right|\right) c_{p} .
$$

Here, $\beta_{p}=k_{p} / k$ and $\beta_{s}=1+\gamma\left|c_{s}\right|^{2} / 4$ are the propagation constants. The coupling parameter is given by $q\left(\left|c_{s}\right|\right) \simeq$ $\exp \left(-k \sqrt{\gamma / 2}\left|c_{s}\right| d\right)$ [12]. The rationale of this functional form is that the lateral tail of the soliton field, which depends on the soliton amplitude $\left|c_{s}\right|$, acts as a source to excite the surface plasmons. Substituting $c_{p, s}=C_{p, s} e^{i \xi}$ and employing the slowly varying amplitude approximation, the following set 

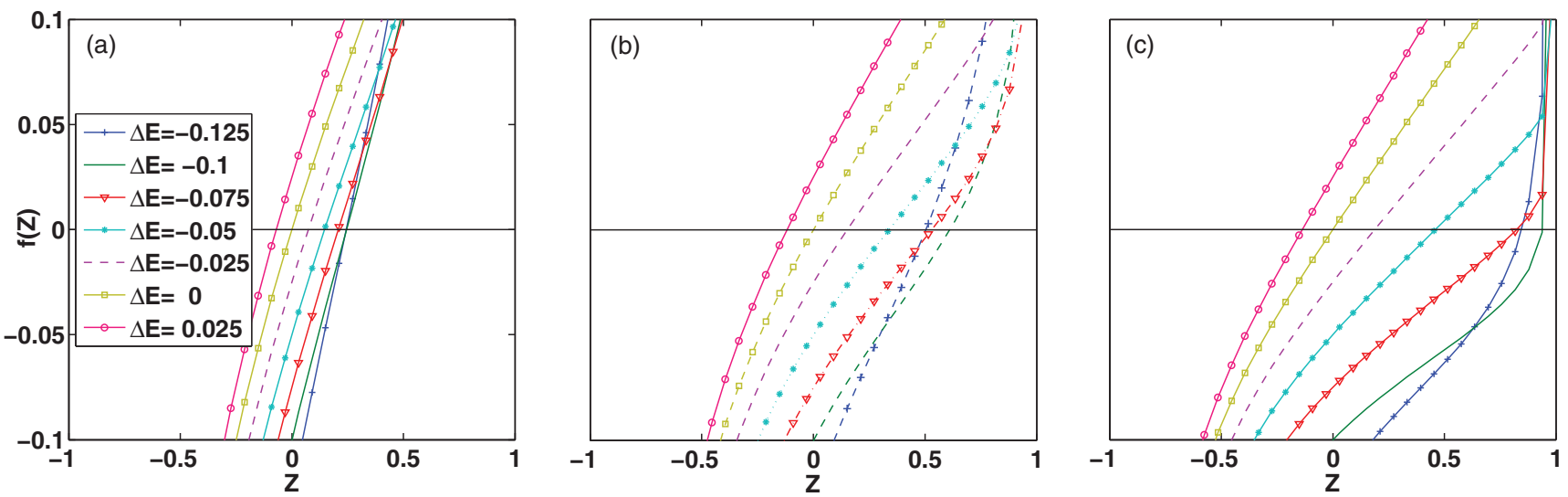

FIG. 1. (Color online) $f(Z)$ Eq. (11) for zero-phase modes plotted at $v_{p}=0.15$ with various values of $\Delta E$ and (a) $k d=3$, (b) $k d=6$, and (c) $k d=12$. The critical points are the roots of $f(Z)=0$.

of equations are obtained for the amplitudes $C_{p, s}$ :

$$
\begin{gathered}
-i \dot{C}_{p}=v_{p} C_{p}-\frac{q\left(\left|C_{s}\right|\right)}{2} C_{p}, \\
-i \dot{C}_{s}=-\frac{q\left(\left|C_{s}\right|\right)}{2} C_{p}+v_{s}\left(\left|C_{s}\right|\right) C_{s} .
\end{gathered}
$$

In Eq. (3), $v_{p} \equiv \beta_{p}-1 \ll 1, v_{s} \equiv \beta_{s}-1 \ll 1$ are the small deviations of the dimensionless propagation constants of surface plasmon and soliton, respectively. The eigenmode analysis indicates that the resonant coupling $q_{\text {res }}=$ $\exp \left[-k d \sqrt{2 v_{p}}\right]$ occurs around $v_{p}=0.2, v_{s}=0.2[12]$.

\section{A. Josephson junction formulation}

Remarkably, Eq. (3) can be cast in analogy to the Josephson junction dynamics by writing $C_{s, p}=\left|C_{s, p}\right| e^{i \phi_{s, p}}$, and introducing the fractional population imbalance $Z=$ $\left(\left|C_{s}\right|^{2}-\left|C_{p}\right|^{2}\right) / N$ and the relative phase difference between the soliton and the surface plasmon $\phi=\phi_{s}-\phi_{p}$ as follows:

$$
\begin{gathered}
\dot{Z}=-q(Z) \sqrt{1-Z^{2}} \sin \phi, \\
\dot{\phi}=\Lambda Z+\Delta E+\frac{q(Z) Z}{\sqrt{1-Z^{2}}} \cos \phi .
\end{gathered}
$$

We set $N=\left(\left|C_{s}\right|^{2}+\left|C_{p}\right|^{2}\right) \equiv 1$, a normalized constant for the isolated system with no population dissipation, and define the parameters $\Lambda \equiv \eta / 2, \Delta E \equiv \eta / 2-v_{p}$, with $\eta=\frac{\gamma N}{4}$. $\Lambda$ is the nonlinearity (i.e., the soliton strength) whereas $\Delta E$ parametrizes the asymmetry between the soliton and surface plasmon states occupied by the photons. The coupling parameter takes the $Z$-dependent form

$$
q(Z)=e^{-k d \sqrt{2 \Lambda(1+Z)}}
$$

An immediate comparison reveals the similarities and differences to the dynamical model of the bosonic Josephson junction (BJJ) in a double-well trap model of two BoseEinstein condensates $[13,17,18]$

$$
\begin{gathered}
\dot{Z}=-\sqrt{1-Z^{2}} \sin \phi, \\
\dot{\phi}=\Lambda Z+\Delta E+\frac{Z}{\sqrt{1-Z^{2}}} \cos \phi .
\end{gathered}
$$

In the BJJ model, $\Lambda$ describes the interatomic interactions and $\Delta E$ is the difference between the zero-point energies of the trapping wells. Both $\Lambda$ and $\Delta E$ are dimensionless parameters scaled by the coupling matrix element [17]. We observe that the DWSP-JJ equations would reduce to that of the BJJ model for a constant coupling parameter $q(Z) \equiv 1$ provided that the other parameters are scaled accordingly.
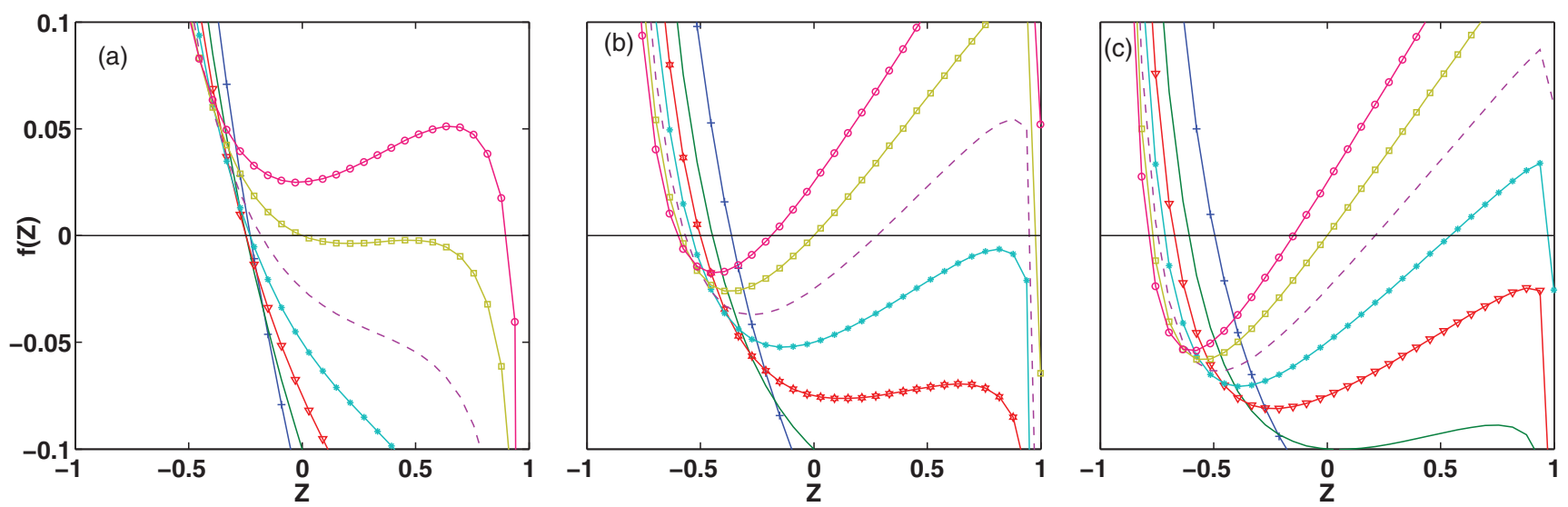

FIG. 2. (Color online) $f(Z)$ Eq. (11) for $\pi$-phase modes plotted at $v_{p}=0.15$ with various values of $\Delta E$ and (a) $k d=3$, (b) $k d=6$, and (c) $k d=9$. The critical points are the $\operatorname{root}(\mathrm{s})$ of $f(Z)=0$. 

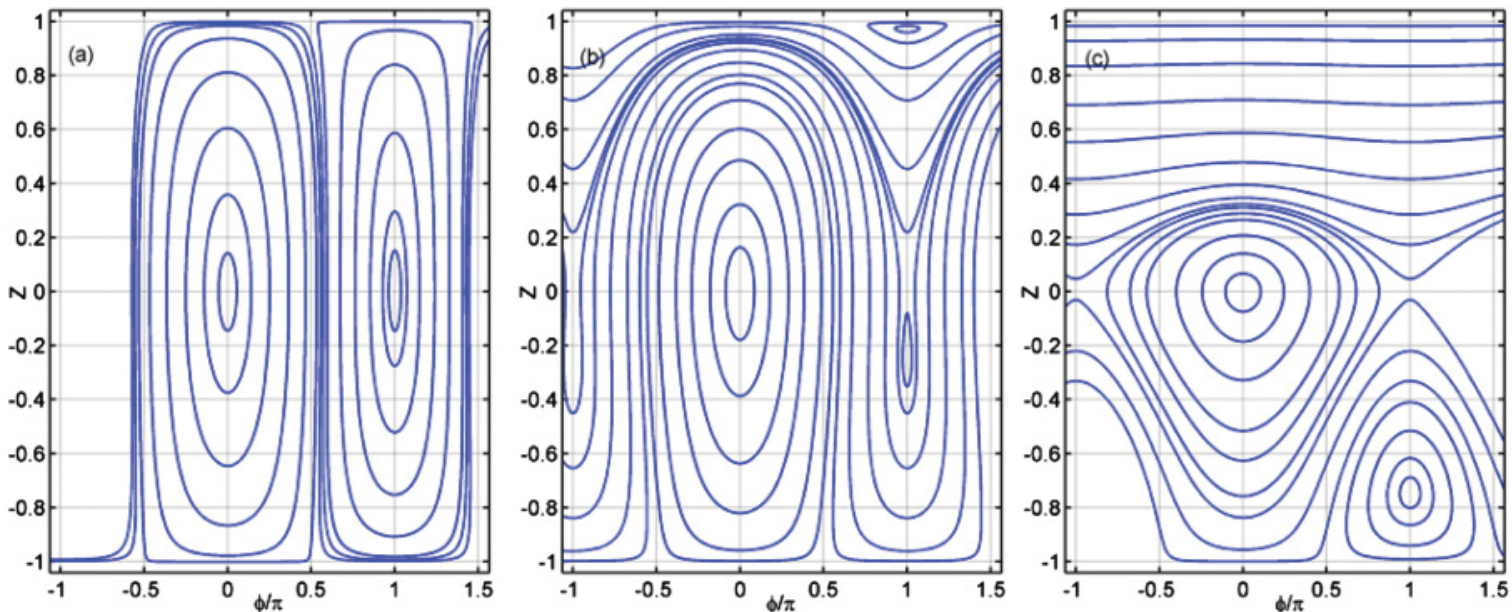

FIG. 3. (Color online) Several phase-space trajectories of the DWSP-JJ system for $\Delta E=0, v_{p}=0.15$, (a) $k d=3$, (b) $k d=6$, and (c) $k d=12$.

It is instructive to present also the case of superconducting Josephson junctions (SJJs) where the dynamical variable is the voltage across the resistive shunt between the two superconductors [19]

$$
\dot{\phi}=\frac{2 e V(t)}{\hbar} .
$$

The SJJ tunneling ac current is given by

$$
\dot{I}=I_{0}(\cos \phi) \dot{\phi} .
$$

The Cooper-pair population imbalance across the SJJ is zero when the SJJ is closed over an external circuit [20]. In the absence of an external circuit, an isolated SJJ can exhibit coherent Cooper-pair oscillations only at very small amplitudes [20,21]. By virtue of the oscillating current, the SJJ is generally discussed in terms of a rigid pendulum analogy. On the other hand, the BJJ dynamics resembles that of a nonrigid pendulum with length-dependent angular momentum $(Z)$ [17].

In the BJJ, the double-well trap is created by a laser field barrier that divides a single trapped condensate in two parts.
Hence, the asymmetry of the wells $(\Delta E)$, as well as the barrier height (i.e., coupling) is controlled externally by the laser field. The coupling can further be modulated temporally by the laser beam.

To this end, we stress that the presence of $Z$-dependent nonlinear coupling in the DWSP-JJ implies fundamentally different dynamical features from the BJJ and SJJ models, particularly in certain parameter regimes, as discussed in the next section.

\section{RESULTS}

We start exploring the dynamical landscape of the DWSP-JJ model first by determining the stationary solutions at fixed points. Equation (4) shows that the fixed points occur at $\left(\phi^{*}=\right.$ $2 \pi)$ or $\left(\phi^{*}= \pm \pi\right)$ with the respective population imbalance values determined from

$$
\left.f(Z)\right|_{Z^{*}}=\Lambda Z^{*}+\Delta E+\frac{q\left(Z^{*}\right) Z^{*}}{\sqrt{1-\left(Z^{*}\right)^{2}}} \cos \left(\phi^{*}\right)=0 .
$$
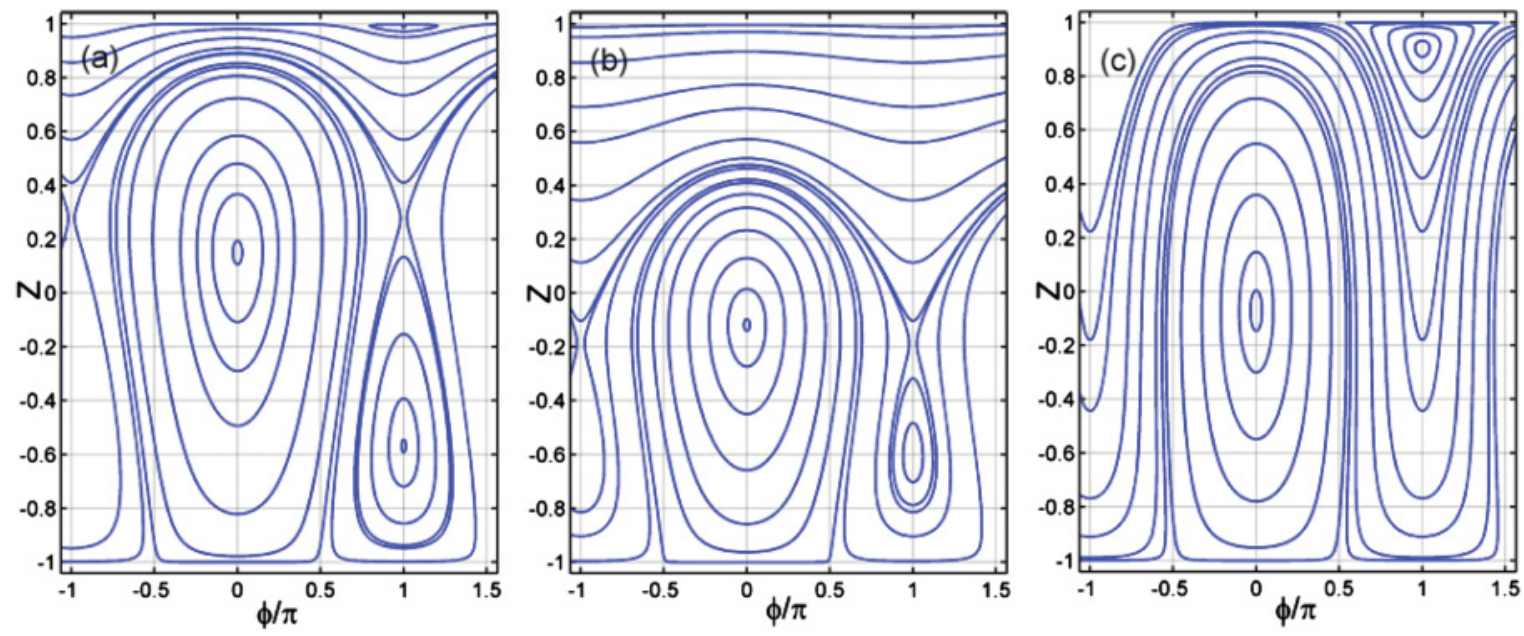

FIG. 4. (Color online) Several phase-space trajectories of the DWSP-JJ system for (a) $\Delta E=-0.025, v_{p}=0.15, k d=6$, (b) $\Delta E=$ $0.025, v_{p}=0.15, k d=6$, and (c) $\Delta E=0.025, v_{p}=0.15, k d=3$. 

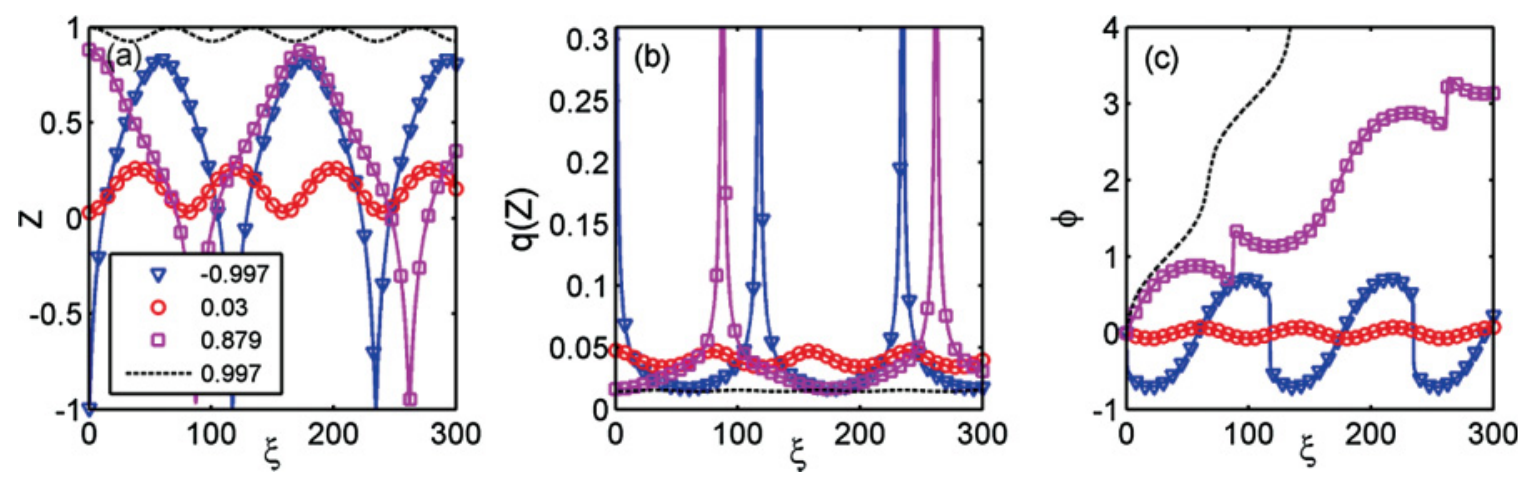

FIG. 5. (Color online) Propagation of (a) $Z$, (b) coupling parameter $q(Z)$, and (c) $\phi$ for $\phi(0)=0$ and for various values of $Z(0)$ indicated in the panel of (a). The model parameters are taken from Fig. 4(a).

We investigate the range $0.05<v_{p}<0.35$, and $0.05<$ $\eta<0.35$ including the resonant coupling $\left(v_{p}=0.2, \eta=0.2\right)$ [12]. Since $v_{p}=\frac{k_{p}}{k}-1$, small values imply $k_{p} \approx k$ regime. The $\eta$ values considered here represent strong nonlinearity when compared with actual physical values. For the scaled distance parameter $k d$ we use the range $3-12$ and discuss the uncoupled limit $(k d \gg 1)$ qualitatively.

\section{A. Fixed points}

The zero-phase modes describe the transfer of energy between the soliton and surface-plasmon states with zero time-average value of the phase. In Figs. 1(a)-1(c) the $f(Z)$ is plotted for different values of $\Delta E$ for $v_{p}=0.15$ and $k d=$ $3,6,12$. For $\Delta E=0$ the fixed point is at $Z=0$, which can also be deduced by inspecting Eq. (11). This is analogous to the symmetric double well of the BJJ model. For $\Delta E<(>) 0$, the fixed point occurs at nonzero fractional population imbalance $Z>(<) 0$. There is a single fixed point for zero-phase modes.

The other mode is the $\pi$-phase mode, where the timeaverage value of phase is $\langle\phi\rangle=\pi$. Up to three fixed points may emerge depending on the model parameters as shown in Fig. 2. Three critical points occur when $\eta$ (nonlinearity) is comparable or larger than $v_{p}$, provided that $v_{p}$ itself is not too small. For smaller values of $v_{p}<0.1$ the $\pi$-phase mode also exhibits a single fixed point.

\section{B. Phase-space analysis}

A concise description of the dynamical behavior can be given by the phase-space representation of Eq. (4). In Figs. 3(a)-3(c), several phase-space trajectories are given for the symmetric case with $v_{p}=0.15$ and $k d=3,6,12$. For $k d=$ 3 [Fig. 3(a)], the zero-phase and $\pi$-phase modes exhibit similar behavior, with rigid pendulum-type closed-orbit oscillations at small amplitudes and anharmonic closed-orbit oscillations at large amplitudes. The running phase trajectories consist of $|Z| \sim 1$ plateaus connected by a population inversion occurring close to $\phi \sim \pm \pi / 2$ points. All these features are similar to the dynamical modes of the BJJ model discussed elsewhere [22].

When the spacing parameter is increased to $k d=6$, we observe a drastic change in the phase-space portrait [Fig. 3(b)]. The anharmonicity of the large-amplitude zero-phase modes become prominent. For the $\pi$ phase, three fixed points appear, of which the $Z^{*} \sim 0.98$ and $Z^{*} \sim-0.25$ are enclosed by bounded trajectories, whereas the $Z^{*}=0$ is an unstable fixed point. In Fig. 3(c), we observe that increasing the $k d$ further $(k d=12)$ results in the decoupling of the system for soliton-dominant initial populations $[Z(0)>0.5]$. This is expected since the coupling parameter $q(Z)$ remains small. On the other hand, a surface-plasmon dominant initial population $[Z(0)<0]$ is still effectively strongly coupled since the coupling parameter can be close to unity even though $k d$ is
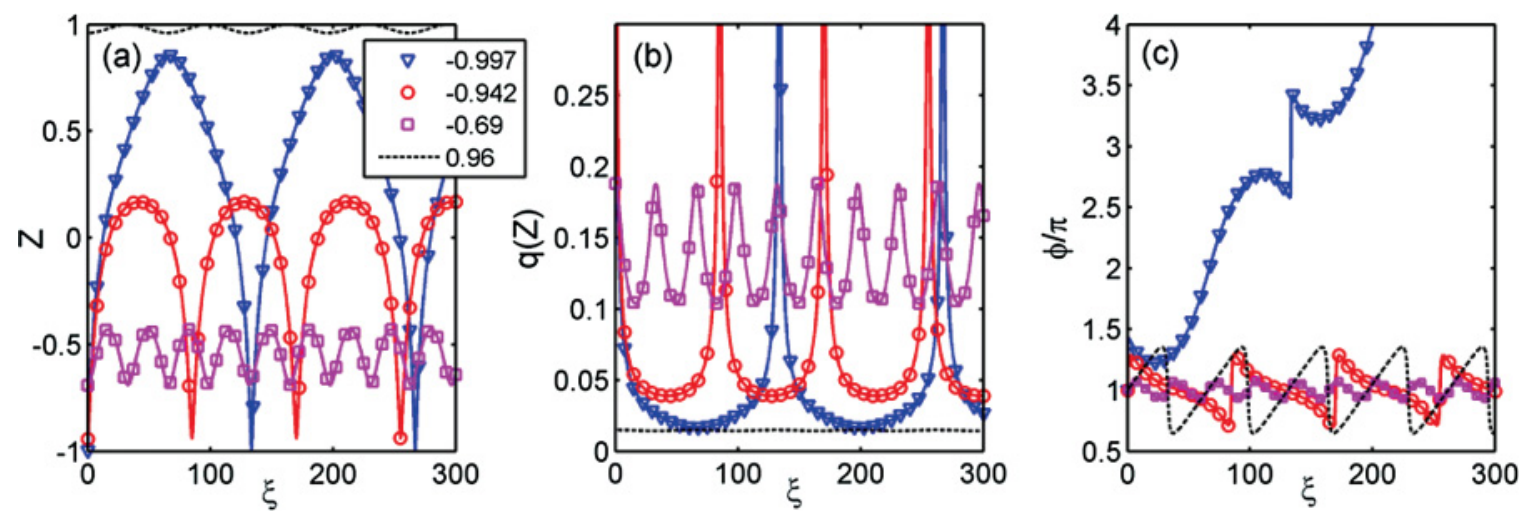

FIG. 6. (Color online) Propagation of (a) $Z$, (b) coupling parameter $q(Z)$, and (c) $\phi$ for $\phi(0)=\pi$ and for various values of $Z(0)$ indicated in the panel of (a). The model parameters are taken from Fig. 4(a). 

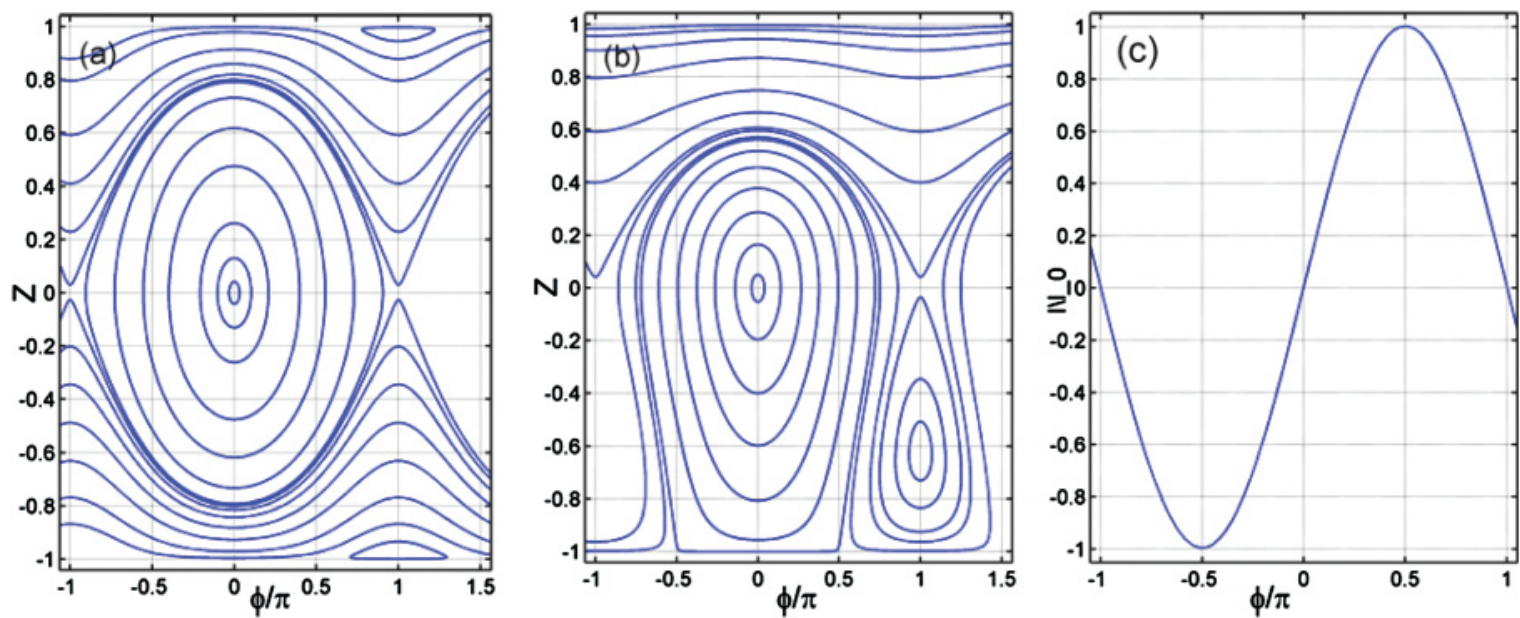

FIG. 7. (Color online) Comparison of the phase-space trajectories of (a) BJJ ( $q \equiv 0.03, \Delta E=0, \Lambda=0.15)$, (b) DWSP-JJ $(k d=6, \Delta E=$ $0, \Lambda=0.15)$, and (c) SJJ models.

large [see Eq. (6)]. The closed orbits of the zero-phase mode widen in phase toward the two unstable fixed points as opposed to being confined around $|\phi|<\pi / 2$. The closed-orbit $\pi$-phase modes occur around $Z^{*} \sim-0.8$. In accordance with Fig. 2(c) another fixed point exists that occurs almost at $Z^{*}=1$ and is not visible in this scale.

The phase trajectories for $(\Delta E \neq 0)$ and fixed $v_{p}=0.15$ are given in Fig. 4. Figure 4(a) is plotted for $\Delta E=-0.025$, $k d=6$. The zero-phase fixed point is located at $Z^{*}=0.19$. The $\pi$-phase closed modes occur around $Z^{*}=-0.6$ and in a narrow region around $Z^{*} \sim 1$. For $\phi(0)=0$ running phase orbits have large amplitude oscillations for $0.81<Z<0.85$ and small oscillations for $0.85<Z$. The phase diagram for $\Delta E=0.025, k d=6$ is shown in Fig. 4(b). The zero phase mode is at $\left(Z^{*}=-0.2\right)$. The $\pi$-phase mode has closed orbits only around $\left(Z^{*}=0.95\right)$. When $k d$ is decreased $(k d=3)$ [Fig. 4(c)] $\phi=\pi$ mode forms closed orbits around $Z^{*}=0.9$.

In the BJJ model, the parameter $\Delta E$ is a measure of the asymmetry between the two trapping states of atoms. Evidently, a nonzero $\Delta E$ induces a population imbalance between the two states. In the DWSP-JJ model, this asymmetry is indicated by the location of the $\phi^{*}=0, Z^{*} \neq 0$ fixed points.

We next investigate the variation of $Z(\xi), q(Z)$, and $\phi(\xi)$ for various trajectories from Fig. 4(a). Figure 5 show the propagation with initial relative phase $\phi(0)=0$ for different initial values of $Z(0)$. As expected from the functional form in Eq. (6), the coupling parameter shows variations commensurate with that of the population imbalance. Small amplitude oscillations [Fig. 5(a), circles and dotted line] have small, almost constant $q(Z)$ whereas large amplitude oscillations (triangle and square) exhibit impulsive behavior of the coupling. Similar observations are present for $\phi(0)=\pi$ in Fig. 6.

We conclude our discussion by a qualitative comparison of the BJJ, DWSP-JJ, and SJJ phase portraits in Figs. 7(a)-7(c), respectively. The normalized SJJ ac current is plotted for a constant junction potential in Eq. (9). A detailed comparison between the SJJ and BJJ was reported in the literature [22]. Here we rather focus on the BJJ and DWSP-JJ phase-space features.
The BJJ model is depicted with an effective coupling constant of $q \sim 0.03$, which is within the dynamic range of the $q(Z)$ of the DWSP-JJ. The phase space of the BJJ is decorated with open- and bounded-phase trajectories that are symmetric with respect to the $Z$ axis. The $\phi(0)=0$ trajectories are bounded for $|Z(0)|<0.8$. The bounded orbits are harmonic for $|Z(0)|<0.5$ and become anharmonic for $0.5<Z(0)<0.8$. The $\phi(0)=\pi$ trajectories are open for $|Z(0)|<0.9$ and become bounded anharmonic orbits for $0.9<|Z(0)|<1$.

In the DWSP-JJ phase portrait plotted with a comparable parameter set [Fig. 7(b)], the asymmetry in $Z$ induced by the dynamical coupling parameter is prominent. Qualitatively speaking, the DWSP-JJ acts like a double-well trap BJJ system, where the barrier height (i.e., the coupling) between the two wells depends on the population of one well (i.e., the soliton amplitude). The bound trajectories are strongly anharmonic except for $|Z(0)<0.2|$. For $\phi(0)=0$, the orbits are closed for all negative $Z(0)$ values and for positive $Z(0)<0.55$. The $\phi(0)=\pi$ states are bounded for $-0.9<Z(0)<0$.

Finally, it should be noted that the present model excludes dissipation effects, which will be tackled extensively in a subsequent work. Nevertheless, the qualitative features demonstrated here are expected to be present under weak dissipative conditions. Both population- and phasedependent dissipation mechanisms are applicable. The effects of phase dissipation are discussed in part in [12].

Even though a proper assessment of experimental feasibility has to take into account the dissipation, we can naively point out some typical parameter values that may serve as guidance. For the infrared regime, the chalcogenide glasses can provide the medium for soliton propagation [23,24]. They have a transmission band $0.7-10 \mu \mathrm{m}$, linear dielectric constant around $\epsilon_{d} \sim 5-8$ and nonlinear index of refraction $n_{2}=10^{-16} \mathrm{~m}^{2} / \mathrm{W}$. For doped silica fibers, nonlinearity will be weaker. The nonlinearity parameter $\gamma$ is related to $n_{2}$ through $\gamma=\frac{n_{2} k}{A_{\text {eff }}}$, where $A_{\text {eff }}$ is the effective core area (of the fiber). For most typical fibers, the nonlinearity parameter has a value $\gamma \sim$ $10^{-3} \mathrm{~W}^{-1} / \mathrm{m}$, but for highly nonlinear fibers $\gamma \sim 10^{-2} \mathrm{~W}^{-1} / \mathrm{m}$ is achievable [25]. Thus, the range of nonlinearity parameter $\eta$ considered here may pose a challenge under experimental 
conditions. Surface plasmons are typically studied on gold or silver surfaces [5]. Depending on the lateral extent of the evanescent surface-plasmon and soliton fields, the $k d$ for weak coupling regime would be typically around $\sim 1-10$ [12]. Overall, we found that for smaller values $(<0.1)$ of $v_{p}$ and $\eta$, the phase-space portrait exhibits single fixed points for $\phi=0$ modes and $\phi=\pi$ modes, respectively. Thus, some of the features presented in the phase space require strong nonlinearity of the dielectric medium.

\section{CONCLUSION}

In this work, we proposed a weakly coupled opticalsoliton and metal surface-plasmon system as another type of
Josephson junction and investigated its dynamical properties. We have found that the coupling parameter that depends on the soliton amplitude allows rich dynamical features, different than that observed in bosonic Josephson junctions. The DWSP-JJ is potentially a convenient system to investigate collective dynamics of photons and surface plasmons, which may reveal classical and quantum plasmonic phenomena.

\section{ACKNOWLEDGMENTS}

We acknowledge the support of the Science and Technology Research Council of Turkey (TUBITAK) under Project No. 111T285. K.G. acknowledges the support of the Turkish Academy of Sciences.
[1] E. A. Stern and R. A. Ferrell, Phys. Rev. 120, 130 (1960).

[2] M. Quinten, A. Leitner, J. R. Krenn, and F. R. Aussenegg, Opt. Lett. 23, 1331 (1998).

[3] S. I. Bozhevilnyi, V. S. Volkov, E. Devaux E, J. Y. Laluet and T. W. Ebbesen, Nature (London) 440, 508 (2006).

[4] S. A. Maier Plasmonics: Fundamentals and Applications (Springer, New York, 2007).

[5] Surface Plasmon Nanophotonics, edited by M. L. Brongersma and P. Kik (Springer, Netherlands, 2007).

[6] R. H. Ritchie, Phys. Rev. 106, 874 (1957).

[7] K. Y. Bliokh, Y. P. Bliokh, V. Freilikher, S. Savelev, and F. Fori, Rev. Mod. Phys. 80, 1201 (2008).

[8] M. Hochberg, T. Baehr-Jones, C. Walker and A. Scherer, Opt. Express 12, 5481 (2004).

[9] F. Liu, Y. Huang, W. Zhang, and J. Peng, Appl. Phys. Lett. 90, 141101 (2007).

[10] R. Zia, A. Chandran, and M. L. Brongersma, Opt. Lett. 30, 1473 (2005).

[11] H. Ditlbacher, N. Galler, D. M. Koller, A. Hohenau, A. Leitner, F. R. Aussenegg, and J. R. Krenn, Opt. Express 16, 10455 (2008).

[12] K. Y. Bliokh, Y. P. Bliokh, and A. Ferrando, Phys. Rev. A 79, 041803(R) (2009).
[13] A. Smerzi, S. Fantoni, S. Giovanazzi, and S. R. Shenoy, Phys. Rev. Lett. 79, 4950 (1997).

[14] S. Raghavan, A. Smerzi, S. Fantoni, and S. R. Shenoy, Phys. Rev. A 59, 620 (1999).

[15] D. E. Chang, A. S. Sorensen, P. R. Hemmer, and M. D. Lukin, Phys. Rev. Lett. 97, 053002 (2006).

[16] E. Feigenbaum and M. Orenstein, Opt. Lett. 32, 674 (2007).

[17] A. R. Davoyan, I. V. Shadrivov, and Y. Kivshar, Opt. Express 17, 21732 (2009).

[18] B. D. Josephson, Rev. Mod. Phys. 46, 251254 (1974).

[19] A. Barone and G. Paterno, Physics and Applications of the Josephson Effect (Wiley, New York, 1982).

[20] M. Tinkham, Introduction to Superconductivity, 2nd ed. (McGraw-Hill, New York, 1996).

[21] I. Marino, S. Raghavan, S. Fantoni, S. R. Shenoy, and A. Smerzi, Phys. Rev. A 60, 487 (1999).

[22] S. Giovanazzi, A. Smerzi, and S. Fantoni, Phys. Rev. Lett. 84, 4521 (2000).

[23] M. Chauvet, G. Fanjoux, K. P. Huy, V. Nazabal, F. Charpentier, T. Billeton, G. Boudebs, M. Cathelinaud, and S.-P. Gorza, Opt. Lett. 34, 1804 (2009).

[24] T. Kaczmarek, Adv. Electr. Telecomm. 1, 59 (2010).

[25] G. P. Agrawal, Nonlinear Fiber Optics, 4th ed. (Academic Press, San Diego, 2007). 